\title{
ESTUDO PRELIMINAR DA ECOLOGIA TRÓFICA DA Sardinella brasiliensis NA COSTA SUDESTE DO BRASIL
}

\author{
SCHNEIDER, F. \& P.R. SCHWINGEL \\ Universidade do Vale do Itajaí, Centro de Ciências Tecnológicas da Terra e do Mar. \\ CP 360, Itajaí (SC), Brasil. \\ email: fabiola.schneider@mailcity.com; schwingel@cttmar.univali.br
}

\begin{abstract}
RESUMO
A ecologia trófica da sardinha verdadeira Sardinella brasiliensis foi estudada na plataforma continental do Brasil, de $22^{\circ} \mathrm{S}$ até $29^{\circ} \mathrm{S}$. A dieta da S. brasiliensis foi analisada qualitativamente e quantitativamente para o outono, inverno e primavera baseado no Índice de Importância Relativa (IRI). A análise volumétrica indireta (chamada método volume individual) foi realizada de forma que os itens alimentares são comparados a formas geométricas, e assim, volumes calculados. A distribuição de comprimento da sardinha variou principalmente entre 170 e $200 \mathrm{~mm}$. As presas apresentaram uma variedade de organismos fitoplanctônicos e zooplanctônicos, incluindo diatomáceas, dinoflagelados, tintinnídeos, copépodos, decápodas e amfípodas. No conteúdo estomacal da $S$. brasiliensis foram identificados 27 taxa diferentes, 11 taxa de fitoplâncton e 16 taxa de zooplâncton. A fração zooplanctônica representou $74,2 \%$ no volume total de presas no outono, $36,8 \%$ no inverno e $99,8 \%$ na primavera. A diatomácea Coscinodiscus spp. e os copépodos foram volumetricamente os taxa mais importantes na dieta. Entre copépodos, o ciclopóida Oncaea sp., o haparticóida Microsetella norvegica, os calanóides Centropages sp., Temora stylifera e Candacia curta foram as espécies mais importantes na dieta. Larvas de Brachyura e o amfípoda Lestrigonus sp. constituíram também itens importantes $S$. brasiliensis foi considerada uma espécie omnívora, pois preda sobre o zooplâncton, mas é capaz de alterar sua estratégia de alimentação para filtração sobre o fitoplâncton e microzooplâncton.
\end{abstract}

Palavras Chave: Ecologia Trófica, Sardinella brasiliensis, Brasil.

\section{A PRELIMINARY STUDY ON THE TROFIC ECOLOGY OF Sardinella brasiliensis OFF SOUTHERN BRAZIL}

\begin{abstract}
The feeding ecology of the brazilian sardine Sardinella brasiliensis was studied on the Brazilian continental shelf, from $22^{\circ} \mathrm{S}$ to $29^{\circ} \mathrm{S}$. Based on the Index of Relative Importance (IRI) the diet $S$. brasiliensis was analysed qualitatively and quantitatively in autumn, winter and spring. An indirect volumetric analysis (called individual volume method) was also performed where food items were classified as geometric bodies and mean volumes were calculated for each food item. The length distribution of sardine in the bulk of the samples varied mainly between 170 and $200 \mathrm{~mm}$. S. brasiliensis prey on a broad variety of phytoplankton and zooplankton organisms, including diatoms, dinoflagellates, tintinnids, copepods, decapods and amphipods. In the stomach contents of $S$. brasiliensis 27 different taxa were identified; 11 phytoplankton and 16 zooplankton taxa. The zooplankton fraction represented $74,2 \%$ of the total prey volume in the sardine stomachs in autumn, $36,8 \%$ in winter and $99,8 \%$ in spring. The diatom Coscinodiscus spp. and copepods were volumetrically the most important taxa in the diet. Among copepods, the cyclopoid Oncaea sp., the harpaticoid Microsetella norvegica, calanoids Centropages sp., Temora stylifera and Candacia curta were the most important species in the diet. Larvae of Brachyura and the amphipod Lestrigonus sp. constituted a large volume in the stomach
\end{abstract}


contents. S. brasiliensis was found to be an omnivore species, which preys on zooplankton, but that is able to switch to filter-feeding on phytoplankton and microzooplankton.

Key Words:Trofic Ecology, Sadinella brasiliensis, Brazil

\section{INTRODUÇÃO}

A sardinha verdadeira (Sardinella brasiliensis, Steindachner 1879) é uma espécie pelágica de águas superficiais da plataforma continental do sudeste do Brasil. Sua ocorrência vai desde o norte do estado do Rio de Janeiro (Cabo de São Tomé, $22^{\circ} S$ ) até o sul do estado de Santa Catarina (Cabo de Santa Marta, $29^{\circ} \mathrm{S}$ ), sendo a espécie mais importante economicamente encontrada na costa de Santa Catarina (Whitehead, 1988) (Fig.1). Na cidade de Itajaí a pesca da sardinha envolve mais de 5 mil empregos diretos e indiretos, computando pescadores, armadores e indústria. A pesca industrial desta espécie é realizada por traineiras de 20 a 30 metros, que utilizam redes de cerco de 700900 metros e altura de 50-60 metros.

A disponibilidade da sardinha para a pesca é caracterizada por grandes flutuações na biomassa, sendo assim, as capturas deste recurso variam de ano para ano não obedecendo padrões definidos (Cergole, 1995). Em 1973 a captura de sardinha chegou a 228.000 toneladas, sendo que a partir de então a pesca se manteve estável até $1986 \mathrm{com}$ capturas entre 100.000 e 170.000 toneladas. A partir de 1987 ocorreu uma drástica queda na captura de sardinha, atingindo apenas 32.000 toneladas no ano de 1990, aumentando para 118.000 toneladas em 1997 (Cergole, 1995; IBAMA, 1998). Para 1999 estimasse a menor captura das últimas três décadas, aproximadamente 25.000 toneladas. A causa destas grandes flutuações de biomassa, que afetam o setor pesqueiro, podem ser as variações das condições oceanográficas (Bakun \& Parrish, 1990) e da oferta de alimento, i.e. variações interanuais das populações de fitoplâncton e zooplâncton.
Com o crescente interesse em administrar melhor esse recurso, é de grande utilidade o conhecimento da ecologia desta espécie (Hyslop, 1980). Estudos sobre a ecologia trófica podem gerar informações importantes para conhecermos o comportamento da sardinha, além disso, sabe-se que a alimentação afeta o crescimento e o recrutamento das populações de peixes (Pitcher \& Hart, 1982; Matsuura, 1996; Zavalla-Camin, 1996). O objetivo principal deste estudo é conhecer a ecologia trófica da sardinha verdadeira desembarcada no porto pesqueiro de Itajaí, identificando variações temporais na sua dieta.

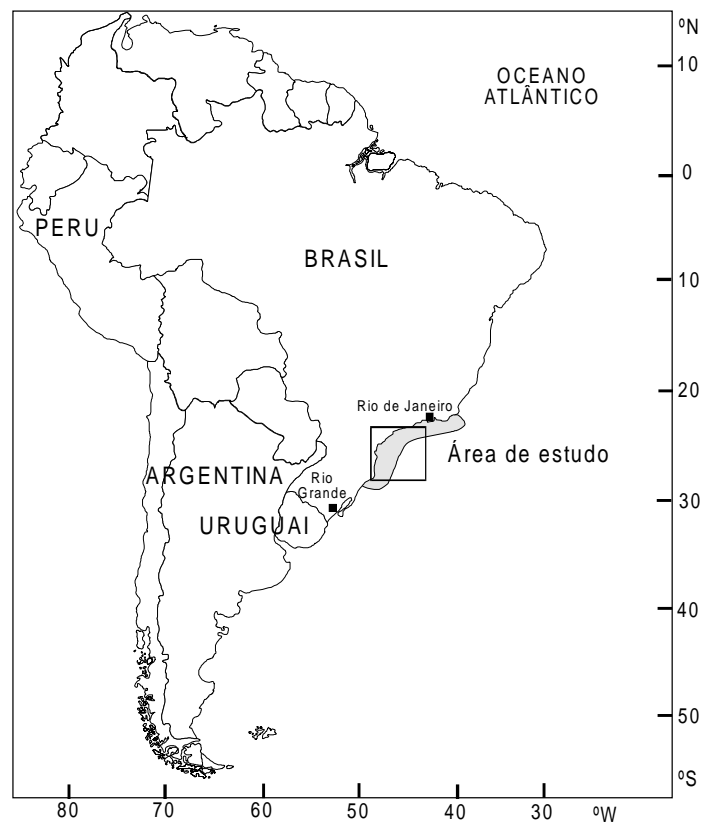

Figura 1: Área de distribuição da Sardinella brasiliensis (mancha escura) e área de estudo (quadrado). 


\section{MATERIAL E MÉTODOS}

As amostras de sardinha utilizadas neste trabalho foram coletadas junto às descargas da frota da indústria de Itajaí, realizadas entre março e dezembro de 1998. No laboratório, os exemplares de sardinha foram medidos e pesados. Depois disso, as porções cardíaca e pilórica do estômago foram retiradas e conservadas em formol $4 \%$ dissolvido em água do mar. Posteriormente os estômagos foram abertos e tiveram seu conteúdo removido. O conteúdo retirado dos estômagos foi filtrado em uma malha de $140 \mathrm{~mm}$. Os itens menores que $140 \mathrm{~mm}$ foram colocados em câmaras de sedimentação e analisados em um microscópio de inversão. Os itens maiores que $140 \mathrm{~mm}$ foram observados em microscópio estereoscópico, sendo que as presas foram identificadas até o menor taxa possível.

Os parâmetros utilizados para a análise foram: porcentagem de ocorrência da presa $(O \%)$, i.e. freqüência de ocorrência; porcentagem numérica da presa (N\%), i.e. abundância; e porcentagem volumétrica da presa $(\mathrm{V} \%)$, i.e. biomassa. O volume das presas foi calculado pela comparação da forma corporal destas e formas geométricas correspondentes, baseado na metodologia aplicada por
Schwingel (1998) para as espécies de fitoplâncton e zooplâncton que ocorrem na costa sudeste do Brasil. Para análise qualitativa e quantitativa da dieta da sardinha e das variações sazonais, foi utilizado o Índice de Importância Relativa (IRI) (Pinkas et al., 1971), a saber:

$$
|R|=\% O(\% N+\% V)
$$

\section{RESULTADOS E DISCUSSÃO}

A sardinha amostrada teve amplitude de $130 \mathrm{~mm}$ até $220 \mathrm{~mm}$, sendo que $90 \%$ dos exemplares estavam na faixa de $170 \mathrm{~mm}$ até $210 \mathrm{~mm}$. Nesta fase preliminar do trabalho foram amostrados 98 estômagos (35 nos meses de outono, 15 no inverno e 48 na primavera). Os resultados foram apresentados na forma de Diagramas IRI, sendo separados em um diagrama para o fitoplâncton e um outro para o zooplâncton, porque se os mesmos fossem apresentados em um mesmo diagrama o fitoplâncton seria sobrestimado devido a sua abundância relativa.

Os resultados mostraram que nos meses de outono o fitoplâncton que correspondeu a $25,8 \%$ do volume do conteúdo alimentar (Fig. 2), teve como maior ocor-
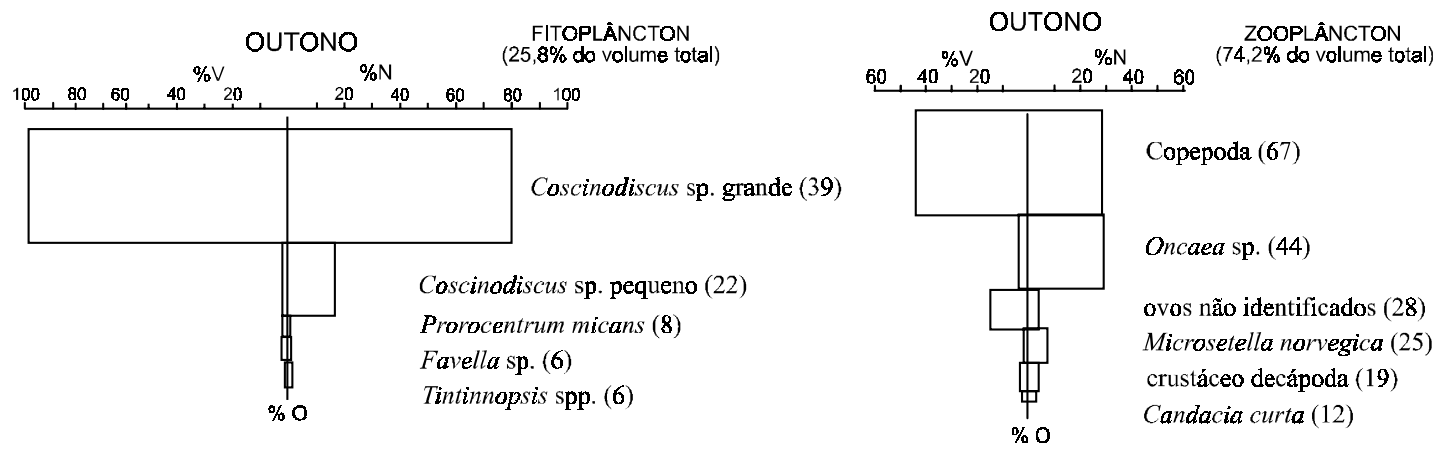

Figura 2: Hierarquia de importância das presas para o zooplâncton e fitoplâncton nos estômagos de $S$. brasiliensis analisados durante o período de outono de 1998. O Diagrama IRI é representado em retângulos de três eixos, onde a porcentagem volumétrica $(\mathrm{V} \%)$ está no eixo $\mathrm{x}$ à esquerda e a numérica no eixo $\mathrm{x}$ à direta e a freqüência de ocorrência (FO\%) está no eixo y e entre parênteses. 
rência o Coscinodiscus spp. grande (39\%), seguido por Coscinodiscus spp. pequeno (22\%), Prorocentrum micans (8\%), Favella sp. (6\%) e Tintinnopsis spp. (6\%). O item Coscinodiscus spp. foi divido em grandes ( $>140 \mathrm{~mm})$ e pequenos $(<140 \mathrm{~mm})$ porque na filtragem a maIha utilizada retinha os organismos maiores, separando-os assim por seus tamanhos. $O$ item que apresentou maior porcentagem volumétrica no fitoplâncton foi 0 Coscinodiscus spp. grande (98\%). O zooplâncton representou $74,2 \%$ do volume da dieta alimentar da sardinha (Fig. 2), sendo o item Copepoda encontrado em $67 \%$ dos estômagos seguido por Oncaea sp. (44\%), ovos não identificados (28\%), Microsetella novergica (25\%), crustáceo decápoda (19\%) e Candacia curta (12\%). Os itens Copepoda e Oncaea sp. obtiveram as maiores freqüências numéricas $(30 \%)$, porém somente os Copepodas tiveram uma freqüência volumétrica significativa (45\%).
Nos meses de inverno o fitoplâncton representou $63,2 \%$ do volume do conteúdo alimentar (Fig. 3) sendo o Coscinodiscus spp. grande a presa mais freqüente (87\%), depois Coscinodiscus spp. pequeno (33\%) e Nitzschia sp. (13\%). O Coscinodiscus spp. grande apresentou uma freqüência numérica de $60 \%$ contra $30 \%$ do Coscinodiscus spp. pequeno, por outro lado, a freqüência volumétrica do Coscinodiscus spp. grande teve dominância sobre as outras espécies atingindo $98 \%$ da biomassa das presas fitoplanctônicas (Fig. 3). Para o zooplâncton, o taxa dos Copepoda continuou a apresentar a maior porcentagem volumétrica $(65 \%) \mathrm{com}$ maior freqüência de ocorrência de $60 \%$. A Oncaea sp. foi a segunda espécie em porcentagem numérica (33\%) e a primeira em freqüência $(74 \%)$, porém apresentou baixa importância volumétrica.

No período de primavera o zooplâncton predominou na alimentação da sardinha re-

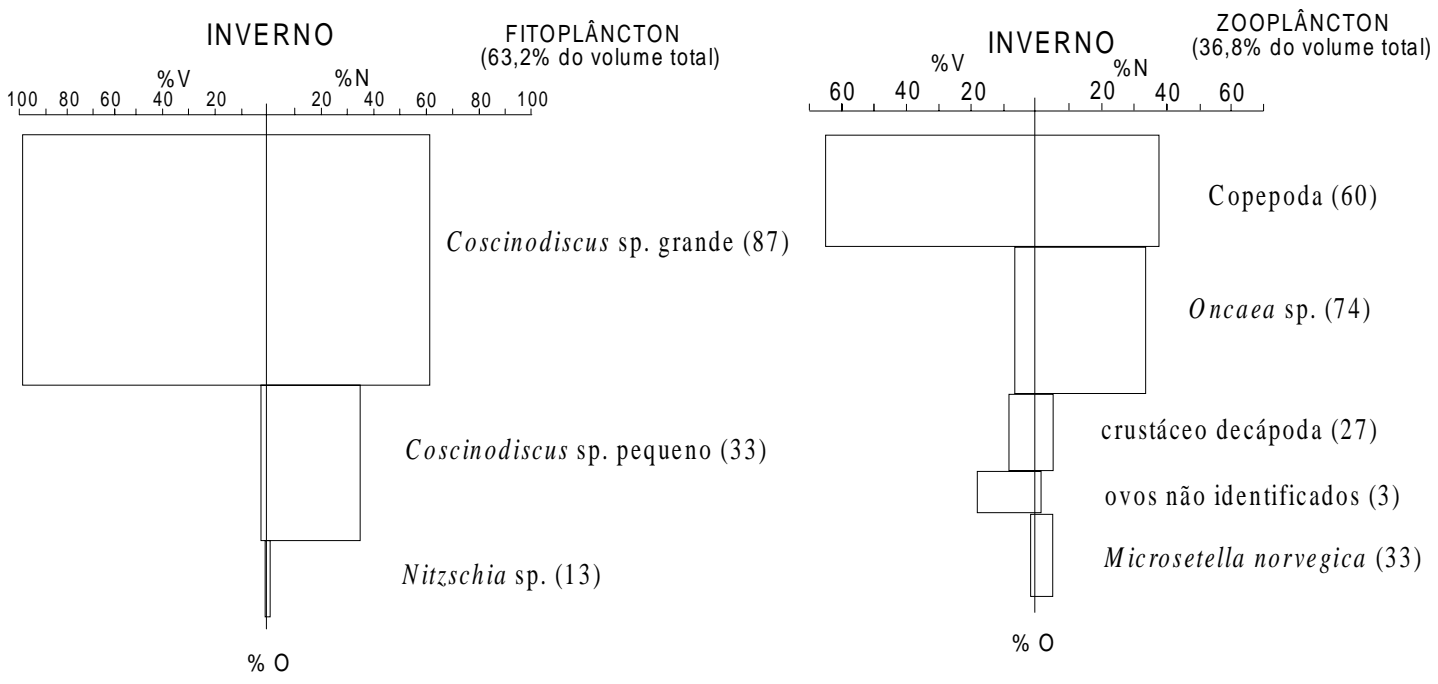

Figura 3: Hierarquia de importância das presas para o zooplâncton e fitoplâncton nos estômagos de $S$. brasiliensis analisados durante o período de inverno de 1998. O Diagrama IRI é representado em retângulos de três eixos, onde a porcentagem volumétrica $(\mathrm{V} \%)$ está no eixo $\mathrm{x}$ à esquerda e a numérica no eixo $\mathrm{x}$ à direta e a freqüência de ocorrência (FO\%) está no eixo y e entre parênteses. 

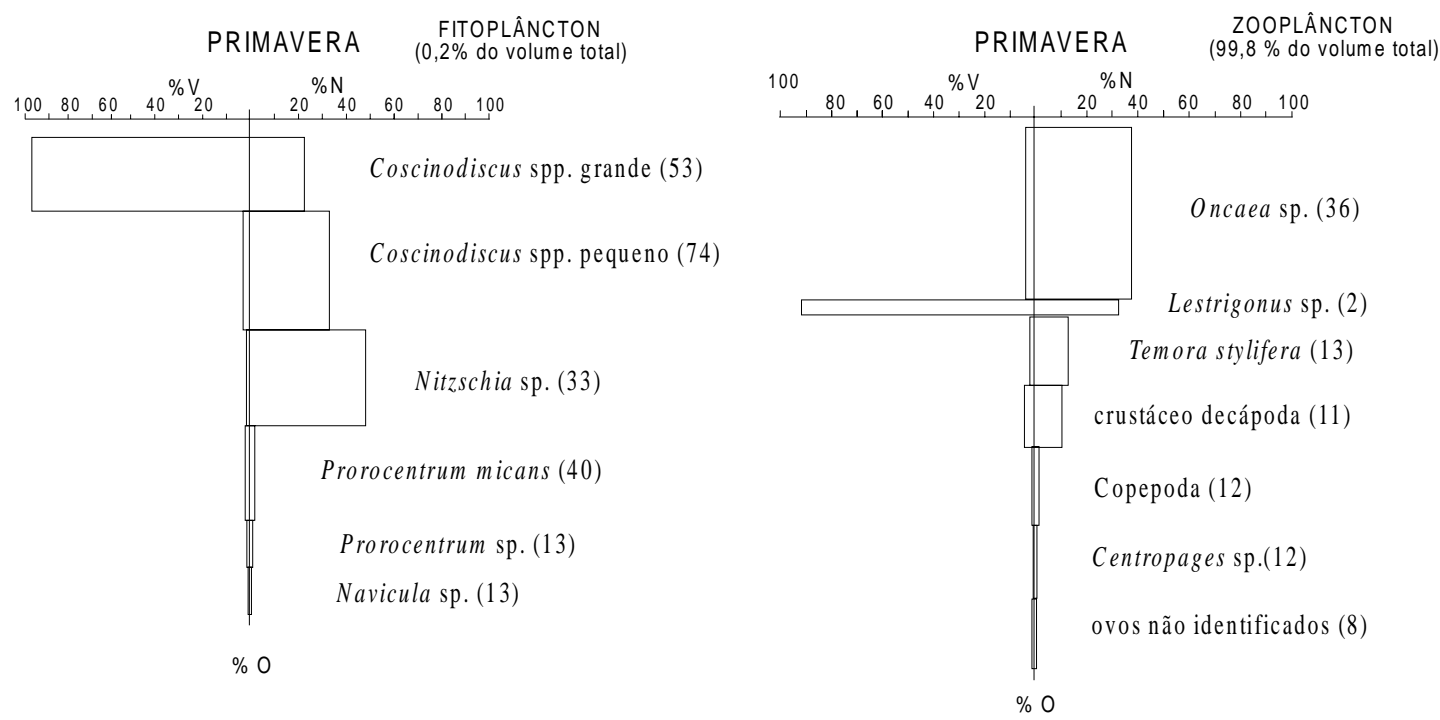

Figura 4: Hierarquia de importância das presas para o zooplâncton e fitoplâncton nos estômagos de $S$. brasiliensis analisados durante o período de primavera de 1998. O Diagrama IRI é representado em retângulos de três eixos, onde a porcentagem volumétrica $(\mathrm{V} \%)$ está no eixo $\mathrm{x}$ à esquerda e a numérica no eixo $\mathrm{x}$ à direta e a freqüência de ocorrência (FO\%) está no eixo y e entre parênteses.

presentando $99,8 \%$ do conteúdo analisado (Fig. 4). A presa com maior freqüência de ocorrência foi o copépodo Oncaea sp. (36\%), seguida por Temora stylifera (13\%), Centropages sp. (12\%) e Copepoda (12\%). O hiperídeo Lestrigonus sp. foi encontrado apenas nos meses de primavera, e apesar de apresentar uma pequena ocorrência $(2 \%)$ teve uma importância volumétrica alta (93\%), sendo esta, a maior biomassa de espécies do zooplâncton na dieta da sardinha verdadeira (Fig. 4). Presas, como os copépodos Temora stylifera e Centropages sp. foram encontradas em outras estações do ano, porém não apresentaram porcentagens de ocorrência tão significativas como na primavera $(13 \%$ e $12 \%$ respectivamente). O fitoplâncton foi pouco representativo neste período $(0,2 \%$ do volume total), sendo que as presas mais freqüentes foram Nitzschia sp. (33\%), Prorocentrum micans (40\%) e Coscinodiscus spp. (53\% e 74\%). O Coscinodiscus spp. gran- de representou quase $100 \%$ do volume fitoplanctônico na primavera.

Em geral, as flutuações sazonais na dieta mostram que no outono e na primavera presas do zooplâncton foram dominantes. No inverno ocorre uma mudança onde o fitoplâncton representa 2/3 do volume estomacal. Goitein (1983) realizou estudos sobre a alimentação da sardinha em Ubatuba, Santos e Cananéia onde constatou também diferenças regionais na predação sobre o fitoplâncton e o zooplâncton. Tais variações na alimentação da $S$. brasiliensis podem estar relacionadas a um efeito sazonal na disponibilidade de alimento, isto já foi verificado para a Engraulis anchoita (Schwingel, 1998) na região sudeste do Brasil. Assim, os resultados preliminares mostram que a sardinha é uma espécie omnívora, alimentando-se sobre o fitoplâncton (produtores) e o zooplâncton (herbívoros e carnívoros). 


\section{BILIOGRAFIA}

BAKUN, A.; PARRISH, R.H. (1990). Comparative studies of coastal pelagic fish reproductive habitats: the Brazilian sardine (Sardinella aurita). J. Cons. int. Explor. Mer, 46: 269-283.

BLAXTER, J.H.S.; HUNTER J.R. (1982). The Biology of the Clupeoid Fishes. In: Advances in Marine Biology, Academic Press, Vol. 20: 1-223.

CERGOLE, M.C. (1995). Stock assessment of the brazilian sardine, Sardinella brasiliensis, of the southeastern coast of Brazil. Sci. Mar., 59 (3-4): 597-610.

GOITEIN, R. (1983). Sardinella brasiliensis (Steindachner, 1879): Estudo sobre a alimentação nas regiões de Ubatuba $\left(23^{\circ} 26^{\prime} S\right)$, Santos $\left(24^{\circ} 02^{\prime} S\right)$ e Cananéia $\left(25^{\circ} 01 ' S\right)$, Brasil. Naturalia, São Paulo, 8: 197-209.

HYSLOP, E.J. (1980). Stomach contents analysis - a review of methods and their application. J. Fish Biol., 17: 411-429.

IBAMA (1998). Informe da Pesca Extrativa Marinha em Santa Catarina 1997.1:14-25.

MATSUURA, Y. (1996). A probable cause of recruitment failure of the brazilian sardine
Sardinella aurita population during the 1974/75 spawnig season. S. Afr. J. mar. Sci. 17:29-35.

PINKAS, L.; OLIPHANT, M.S.; IVERSON, I.L.R. (1971). Food habits of albacore, bluefin tuna, and bonito in California waters. Fish. Bull. 152: 139 p.

PITCHER, T.J.; HART, P.J.B. (1982). Fisheries Ecology. Ed. Croom Helm., London: 414p. ROSSI-WONGTSCHOWSKI, C.L.D.B.; SACCARDO, S.A.; VAZZOLER, A.E.A. (1991). Características biológicas dos cardumes de sardinha e relação com as condições oceanográficas na região sudeste do Brasil (outubro/novembro de 1988). Publicação esp. Inst. oceanogr., S. Paulo, (8): 31-39.

SCHWINGEL, P.R. (1998). Feeding Ecology of Engraulis anchoita (Hubbs \& Marini $1935)$ in Brasilian Waters $\left(22^{\circ} \mathrm{S}\right.$ to $\left.34^{\circ} \mathrm{S}\right)$. Tese de Doutorado, Universidade de Hamburg, Alemanha: $204 \mathrm{p}$.

WHITEHEAD, P.J.P. (1988). FAO species catalogue. Vol. 7. Part I, Pt.1:303 p.

ZAVALA-CAMIN, L. A. (1996). Introdução aos Estudos sobre alimentação natural em peixes. Ed. Nupélia: 129p. 